\title{
OPTICAL FLARES OF CYGNUS X-1
}

\author{
N.G. BOCHKAREV, E.A. KARITSKAYA AND V.M. LYUTY \\ Sternberg Astronomical Institute, Moscow, 119899, Russia \\ Institute of Astronomy, Pyatnitskaya st., 48, Moscow, Russia
}

Photoelectric observations of Cyg X-1 in 1996-97 fulfilled in Tien-Shan, Kazakhstan (WBVR), Mt. Maidanak, Uzbekistan (UBVR) and in Crimea, Ukraine (UBV) have detected unusual optical flare activity. During MayAugust 1996 Cyg X-1 was in high soft X-ray state and after August 9, 1996 it returned to its ordinary state but with some evidences of X-ray activity.

Karitskaya et al. (1997) have registered two large flares (June 2, 1996 $=$ JD 2450267 and October 9, $1996=$ JD 2450366) in U, B, V and R bands with the amplitudes about 0.04 magnitude and durations $\geq 2$ days and $\geq 1$ day. The amplitudes of the flares were approximately of the same magnitude in all the bands. According to X-ray AMS/RXTE data (http://space.mit.edu/XTE) the flares coinsided in time with $20 \% \mathrm{X}$-ray 3-12 keV dips (Fig.1, upper dot set). The power of the flares is approximately $10^{36} \mathrm{erg} / \mathrm{s}$ (about $3 * 10^{35} \mathrm{erg} / \mathrm{s}$ per band).

On May 14, 1997 V.Lyuty and G.Zaitseva detected a very powerful single flare. Similar flares were not previously observed during more than 25 years of Cyg X-1 optical observations. During 2.5 hours long observations Cyg X-1 was $0.12 \mathrm{mag}$ brighter than expected from the regular orbital light curve in bands $U$ and $B$ and only 0.02 mag brighter in $V$ band. Preceding and following nights there was no noticeable brightness deviations. The power of the flare was about $10^{36} \mathrm{erg} / \mathrm{s}$ in each $U$ and $B$ bands.

The colors of the flare of May 14, 1997 correspond to optically thin hydrogen gas region with the temperature $\mathrm{T}=(1-3) * 10^{4} \mathrm{~K}$. Here the hydrogen lines dominate in R and B bands. The He II 4686 line is in B band. There are no strong permitted lines in $\mathrm{V}$ band and the luminosity of Pashen continuum can be small. This gas can be ejected by a quasipoint explosion with a velocity $\mathrm{v}=1500 \mathrm{~km} / \mathrm{s}$. The gas parameters are as follows: $\mathrm{T}=(10-30) * 10^{4} \mathrm{~K}$; volume emission measure $\mathrm{MV}=n_{e} * V \simeq 10^{61} \mathrm{~cm}^{-3}$; radius $\mathrm{R} \sim 3 * 10^{12} \mathrm{~cm}$; electron number density $n_{e} \sim 3 * 10^{11} \mathrm{~cm}^{-3}$; mass $\mathrm{M} \sim 3 * 10^{-8}$ solar mass; kinetic energy $\mathrm{E} \sim 10^{40} \mathrm{erg}$. 


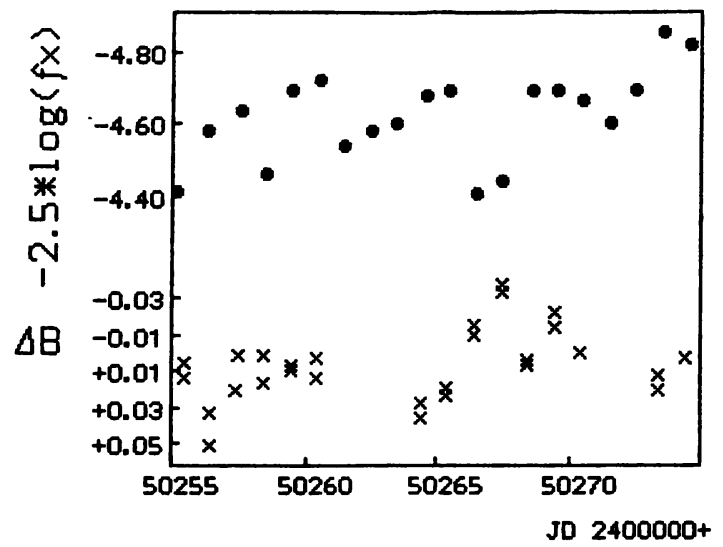

Figure 1. Optical flare observed June 2, 1996 in Mt.Maidanak observatory (Karitskaya et al., 1997). Comparison of AMS/RXTE 3-12 keV X-ray data (upper part of the figure) with the optical light curve in $\mathrm{V}$ band (lower part of the figure). $\mathrm{X}$-ray data are given in the logarithmic scale of magnitudes. For $V$ band deviation from orbital double wave curve is shown. Residuals of the daily averaged X-ray data are less than circle dimentions.

Hydrodynamic time of the outflow is only 5-6 hours. Therefore this outflow was to occur several hours before the observations were started and disappeared during the next $0.5-1$ day.

The June 2, 1996 and October 9, 1996 flares were smaller and emitted in all the bands. In these cases the gas is assumed to be of higher density. It can be ff-emission of gas with $\mathrm{MV} \simeq 10^{61} \mathrm{~cm}^{-3}$. It is probably the emission from optically thick gas balk on the outer part of the accretion disk. This "hot spot" can be originated as a result of interaction of the disk matter with the gas inflowing into the disk. The existense of similar gas balk on the outer part of Cyg X-1 accretion disk has been suggested by Kemp (1980) for interpretation of optical linear polarization orbital variations. Flare luminosity can be explained by re-radiation of X-ray emission of the central part of the accretion disk by the gas balk with $R \simeq 1 / 3$ of the disk radius. The $\mathrm{X}$-ray dips arise as a result of eclipsing of $\mathrm{X}$-ray source by the outer part of the gas balk, where the column density of gas $N \simeq 10^{23} \mathrm{~cm}^{-2}$ is lower then $\mathrm{N}$ following from MV for main part of the "spot".

The work is supported by RBRF grant 96-02-18044 and the program "Monitoring of Unique Astrophysical Objects" Russian Ministry of Science.

\section{References}

Karitskaya, E.A., Goranskij, V.P. and Grankin, K.N. (1997) Properties of Optical Brightness Variations of Cyg X-1 in 1995- 1996 Including X-ray Hard and Soft States, JENAM-97, Thessaloniki, Greece; (in preparation to Pisma v Astron. Zh.) Kemp, J. (1980) On the Allowed Size of an Accretion Disk in Cygnus X-1, Astrophysical Journal, 235 pp. $595-602$ 